\title{
Performance of amplify-and-forward cooperative networks with differential unitary space time coding
}

\author{
Woong Cho
}

Published online: 25 November 2010

(C) The Author(s) 2010. This article is published with open access at Springerlink.com

\begin{abstract}
In wireless communications, multi-input multioutput technology has been appreciated for its high transmission rate and diversity gain. These can also be exploited in cooperative networks by using network nodes as virtual antenna arrays. In this paper, we analyze the performance of cooperative networks with differential unitary spacetime modulation under high signal-to-noise ratio. Relaying protocol is based on amplify-and-forward. The effect of relay location and energy allocation as well as the comparison with a conventional cooperative network is also addressed.
\end{abstract}

Keywords Cooperative networks - MIMO technologies · Differential modulations · Performance analysis

\section{Introduction}

Multi-input multi-output (MIMO) scheme in communication systems enhances the system performance and capacity [12]. However, a practical implementation is cumbersome due to the antenna packing constraint especially when small-sized devices are adopted. Cooperative networks provide spatial diversity gain by creating virtual antenna arrays without imposing antenna packing limitation [8]. To bypass channel state information (CSI) and reduce hardware complexity, a space time coding (STC) scheme with noncoherent modulation has been introduced to cooperative networks $[7,9,15,16]$.

W. Cho $(\square)$

Electronics and Telecommunications Research Institute (ETRI), Daejeon 305-700, Republic of Korea

e-mail: woongcho@etri.re.kr
In this paper, we will consider cooperative networks employing the differential unitary space time code (DUSTC) which does not require CSI both at the relay and destination nodes. The DUSTC is generated at the source node, and its amplified signal is transmitted to the destination node over a common relay-destination channel, which is referred as the amplify-and-forward (AF) relaying protocol. In the literature, differential modulations for cooperative networks have been considered in e.g., [3, 4, 17]. However, unlike the STC-based approach here, existing differential schemes use relays on an individual basis instead of using them jointly. We consider sequential transmission of STC using relay nodes with same frequency band at the source-relays and relays-destination links, which is different from the existing literature in $[7,9$, 15]. Based on the DUSTC signaling scheme, the performance of cooperative networks is analyzed in the form of codeword error rate (CER) under the high signal-to-noise ratio (SNR) assumption. Resource allocation has been considered as an important research topic in cooperative networks, and it has been shown that the location of relays is critical on the error performance [2, 4, 3, 14]. In this paper, we consider the effect of relay locations in cooperative networks including energy allocation. In addition, our results are compared with the cooperative networks which send the signal with a repetitive manner, namely the conventional cooperative networks, as in [8, 4, 17].

The rest of this paper is organized as follows. The signal representation and demodulation rule are described in Sect. 2. In Sect. 3, the upper bound of CER is derived. Simulations, discussions and comparisons are presented in Sect. 4, and concluding remarks are given in Sect. 5 .

Notation: We use bold upper case letters to denote matrices. $\boldsymbol{I}_{N}$ represents an $N \times N$ identity matrix and $\operatorname{diag}\left\{a_{1}, a_{2}, \ldots, a_{N}\right\}$ stands for a diagonal matrix with 
$\left[a_{1}, a_{2}, \ldots, a_{N}\right]$ on its diagonal. We use $(\cdot)^{\mathcal{H}}$ for Hermitian, $\mathcal{C N}\left(\mu, \sigma^{2}\right)$ for the complex Gaussian distribution with mean $\mu$ and variance $\sigma^{2},\|\cdot\|$ for Frobenius norm, and $:=$ for "is defined as".

\section{System model}

Consider a network setup with one source node $s, L$ relay nodes $\left\{r_{k}\right\}_{k=1}^{L}$, and one destination node $d$, as depicted in Fig. 1. We assume that each node works in half-duplex mode. At the source node the DUSTC is generated using the diagonal design with the cyclic construction [6]. Notice that each diagonal element of the codeword has equallyspaced phases like the standard symbol-by-symbol PSK signaling with the number of phases increasing as $L$ increases. We adopt the AF relaying protocol in which the relay nodes simply amplify the received signal from the source node and forward it to destination node.

During the first $L$ time slots of a transmission, the diagonal entries of the DUSTC symbol block are broadcast to the relays by sequential manner. Then, the $k$ th relay node amplifies the corresponding $k$ th diagonal element of the STC matrix, i.e., only the corresponding diagonal element of STC matrix is amplified. At the $k$ th relay node, the other row elements which are not corresponding to the $k$ th diagonal element are disregarded. These amplified signals are then transmitted via a common relays-destination link during the following $L$ time slots. Notice that the STC signal is transmitted sequentially by only considering time division. However, distributed differential schemes in [7, 9, 15] transmits signal using both time and frequency, i.e., space and time.

Denote the $n$th differentially encoded signal block from the source as $\boldsymbol{X}_{n}^{s}:=\boldsymbol{X}_{n-1}^{s} \boldsymbol{V}^{\left(Q_{n}\right)}$ with $\boldsymbol{X}_{0}^{s}=\boldsymbol{I}_{L}$, where $\boldsymbol{V}^{\left(Q_{n}\right)}$ is an $L \times L$ diagonal unitary matrix, $Q_{n} \in\{0,1, \ldots, M-1\}$ with

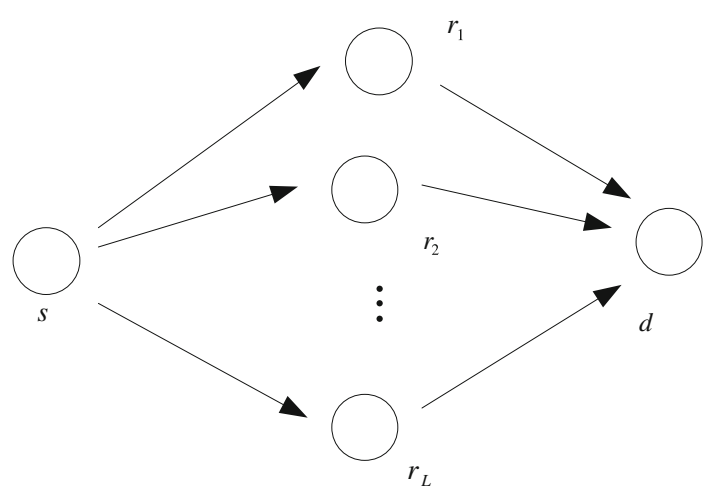

Fig. 1 A wireless system with L relays
$M=2^{\eta L}$, and $\boldsymbol{I}_{L}$ is an $L \times L$ identity matrix, where $\eta$ represents the data rate of the original information which we set to 1. The matrix $\boldsymbol{V}^{\left(Q_{n}\right)}$ has the form $\boldsymbol{V}^{\left(Q_{n}\right)}=\boldsymbol{V}_{1}^{Q_{n}}$ with (see [6])

$\boldsymbol{V}_{1}=\left(\begin{array}{ccc}e^{j(2 \pi / M) u_{1}} & 0 & \cdots \\ 0 & \ddots & 0 \\ 0 & \cdots & e^{j(2 \pi / M) u_{L}}\end{array}\right)$,

where $u_{l} \in\{0, \ldots, M-1\} ; l=\{1, \ldots, L\}$. Then, the $n$th received signal block at the relays is given by

$\boldsymbol{Y}_{n}^{r}=\sqrt{\mathcal{E}_{s}} \boldsymbol{H}_{n}^{r} \boldsymbol{X}_{n}^{s}+\boldsymbol{Z}_{n}^{r}$

where $\mathcal{E}_{s}$ is the energy per symbol at the source, $\boldsymbol{H}_{n}^{r}:=$ $\operatorname{diag}\left\{h_{n}^{s r_{1}}, h_{n}^{s r_{2}}, \ldots, h_{n}^{s r_{L}}\right\}$ is the channel matrix between the source and relays, and $\boldsymbol{Z}_{n}^{r}:=\operatorname{diag}\left\{z_{n}^{s r_{1}}, z_{n}^{s r_{2}}, \ldots, z_{n}^{s r_{L}}\right\}$ is the noise matrix at the relays. Let us denote the $n$th transmitted signal block from the relays as $\boldsymbol{X}_{n}^{r}$, then the corresponding received signal block at the destination is given by

$\boldsymbol{Y}_{n}^{d}=\boldsymbol{E}_{r}^{1 / 2} \boldsymbol{H}_{n}^{d} \boldsymbol{X}_{n}^{r}+\boldsymbol{Z}_{n}^{d}$,

where $\boldsymbol{E}_{r}:=\operatorname{diag}\left\{\mathcal{E}_{r_{1}}, \mathcal{E}_{r_{2}}, \ldots, \mathcal{E}_{r_{L}}\right\}$ is the energy per symbol at the relays, $\boldsymbol{H}_{n}^{d}:=\operatorname{diag}\left\{h_{n}^{r_{1} d}, h_{n}^{r_{2} d} \ldots, h_{n}^{r_{L} d}\right\}$ is the channel matrix between the relays and destination, $\boldsymbol{Z}_{n}^{d}:=$ $\operatorname{diag}\left\{z_{n}^{r_{1} d}, z_{n}^{r_{2} d}, \ldots, z_{n}^{r_{L} d}\right\}$ is the noise matrix at the destination, and the amplified signal block at the relays, $\boldsymbol{X}_{n}^{r}$, can be represented as

$\boldsymbol{X}_{n}^{r}=\boldsymbol{A} \boldsymbol{Y}_{n}^{r}$,

where $\boldsymbol{A}:=\operatorname{diag}\left\{A_{r_{1}}, A_{r_{2}}, \ldots, A_{r_{L}}\right\}$ is the amplification matrix with $A_{r_{k}}$ being the amplification factor at the $k$ th relay. To maintain a constant average power at each relay output, the amplification factor is given by

$A_{r_{k}}=\left(\sigma_{s r_{k}}^{2} \mathcal{E}_{s}+\mathcal{N}_{0}\right)^{-1 / 2}, \quad k=1,2, \ldots, L$.

Then, using the differential modulation, the received signal block at the destination can be represented as

$\boldsymbol{Y}_{n}^{d}=\tilde{\boldsymbol{H}}_{n} \boldsymbol{X}_{n}^{s}+\tilde{\boldsymbol{Z}}_{n}^{d}=\boldsymbol{Y}_{n-1}^{d} \boldsymbol{V}_{n}^{(m)}+\tilde{\boldsymbol{Z}}_{n}^{\prime d}$,

where $\tilde{\boldsymbol{H}}_{n}=\sqrt{E_{s}} \boldsymbol{E}_{r}^{1 / 2} \boldsymbol{A} \boldsymbol{H}_{n}^{d} \boldsymbol{H}_{n}^{r}, \tilde{\boldsymbol{Z}}_{n}^{d}=\boldsymbol{E}_{r}^{1 / 2} \boldsymbol{A} \boldsymbol{H}_{n}^{d} \boldsymbol{Z}_{n}^{r}+\boldsymbol{Z}_{n}^{d}$, and $\tilde{\boldsymbol{Z}}_{n}^{\prime d}=\tilde{\boldsymbol{Z}}_{n}^{d}-\tilde{\boldsymbol{Z}}_{n-1}^{d} \boldsymbol{V}_{n}^{(m)}$.

Since transmission signal is based on the differential space-time code, we can apply the corresponding spacetime differential demodulation. Then, the maximum likelihood (ML) differential demodulation rule [6], given $\boldsymbol{X}_{n}^{s}=\boldsymbol{X}_{n}^{m}$, is

$\hat{Q}_{n}=\arg \max _{m \in\{0,1, \ldots, M-1\}}\left\|\boldsymbol{Y}_{n-1}^{d}+\boldsymbol{Y}_{n}^{d} \boldsymbol{V}_{n}^{(m)^{\mathcal{H}}}\right\|$,

which represents a general decision for DUSTC.

Throughout this paper, all fading coefficients are assumed to be independent and all noise components are independent and identically distributed (i.i.d) with $h_{n}^{i j} \sim \mathcal{C N}\left(0, \sigma_{i j}^{2}\right)$ and 
$z_{n}^{i j} \sim \mathcal{C N}\left(0, \mathcal{N}_{0}\right), i, j \in\left\{s, r_{k}, d\right\}$. Then, the received instantaneous signal-to-noise ratio (SNR) between the transmitter $i$ and the receiver $j$ is $\gamma_{i j}=\left(\left|h_{n}^{i j}\right|^{2} \mathcal{E}_{i}\right) / \mathcal{N}_{0}$, and the average received SNR is $\bar{\gamma}_{i j}=\left(\sigma_{i j}^{2} \mathcal{E}_{i}\right) / \mathcal{N}_{0}$.

\section{Performance analysis}

In this section, we will analyze the error performance of the cooperative system employing the DUSTC. Under high SNR assumption, the upper bound of CER, $P_{e}$, will be derived using the proposed scheme. The CER can be found by calculating the pairwise CER between the source and destination. Let us first consider the covariance matrix of the aggregate noise $\tilde{\boldsymbol{Z}}_{n}^{\prime d}$ in Eq. 6, which bears the form $\operatorname{diag}\left\{\sigma_{h_{1, \text { eff }}}^{2}, \sigma_{h_{2, \text { eff }}}^{2}, \ldots, \sigma_{h_{L, \text { eff }}}^{2}\right\}$, where the corresponding $k$ th diagonal entry of the covariance matrix is given by

$\sigma_{h_{k, e f f}}^{2}=2 \mathcal{N}_{0}\left(\mathcal{E}_{r_{k}} A_{r_{k}}^{2} \sigma_{r_{k} d}^{2}+1\right), \quad k=1,2, \ldots, L$.

To normalize the aggregate noise variance, let us define the matrix $\boldsymbol{G}:=\operatorname{diag}\left\{g_{1}, g_{2}, \ldots, g_{L}\right\}$ with $g_{k}=$ $\left(\mathcal{E}_{r_{k}} A_{r_{k}}^{2} \sigma_{r_{k} d}^{2}+1\right)^{-1 / 2}$. Then, by multiplying $\boldsymbol{G}$ with the received signal block at the destination, we can rewrite Eq. 6 as

$\boldsymbol{Y}_{n}^{d} \boldsymbol{G}=\boldsymbol{Y}_{n-1}^{d} \boldsymbol{V}_{n}^{(m)} \boldsymbol{G}+\tilde{\boldsymbol{Z}}_{n}^{\prime d} \boldsymbol{G}$,

or equivalently, we have

$\tilde{\boldsymbol{Y}}_{n}^{d}=\boldsymbol{Y}_{n-1}^{d} \tilde{\boldsymbol{V}}_{n}^{(m)}+\tilde{\boldsymbol{Z}}_{n}$

where $\tilde{\boldsymbol{Y}}_{n}^{d}=\boldsymbol{Y}_{n}^{d} \boldsymbol{G}, \tilde{\boldsymbol{V}}_{n}^{(m)}=\boldsymbol{V}_{n}^{(m)} \boldsymbol{G}$, and $\tilde{\boldsymbol{Z}}_{n}=\tilde{\boldsymbol{Z}}_{n}^{\prime d} \boldsymbol{G}$. Then, the conditional CER can be calculated using Eq. 10, which is given by

$$
\begin{aligned}
P\left[\tilde{\boldsymbol{V}}_{n} \rightarrow \tilde{\boldsymbol{V}}_{n}^{\prime} \mid \boldsymbol{Y}_{n-1}\right] & =Q\left(\sqrt{D_{c}^{2}\left(\tilde{\boldsymbol{V}}_{n}, \tilde{\boldsymbol{V}}_{n}^{\prime}\right) / 4 \mathcal{N}_{0}}\right) \\
& \leq \exp \left[-D_{c}^{2}\left(\tilde{\boldsymbol{V}}_{n}, \tilde{\boldsymbol{V}}_{n}^{\prime}\right) / 8 \mathcal{N}_{0}\right],
\end{aligned}
$$

where $D_{c}^{2}\left(\tilde{\boldsymbol{V}}_{n}, \tilde{\boldsymbol{V}}_{n}^{\prime}\right)=\operatorname{tr}\left\{\boldsymbol{Y}_{n-1}\left(\boldsymbol{V}_{n}-\boldsymbol{V}_{n}^{\prime}\right) \boldsymbol{G} \boldsymbol{G}^{\mathcal{H}}\left(\boldsymbol{V}_{n}-\boldsymbol{V}_{n}^{\prime}\right)^{\mathcal{H}}\right.$ $\left.\boldsymbol{Y}_{n-1}^{\mathcal{H}}\right\}$. Using the relationship $\boldsymbol{Y}_{n}^{d} \approx \tilde{\boldsymbol{H}}_{n} \boldsymbol{X}_{n}^{s}$ at high SNR (c.f. Eq. 6), the code distance can be approximated as

$D_{c}^{2}\left(\tilde{\boldsymbol{V}}_{n}, \tilde{\boldsymbol{V}}_{n}^{\prime}\right) \approx \operatorname{tr}\left\{\left(\boldsymbol{H}_{n}^{d} \boldsymbol{H}_{n}^{r}\right) \Delta_{e}\left(\boldsymbol{H}_{n}^{d} \boldsymbol{H}_{n}^{r}\right)^{\mathcal{H}}\right\}$,

where $\Delta_{e}=\mathcal{E}_{s} \boldsymbol{E}_{r}^{1 / 2} \boldsymbol{X}_{n-1}\left(\boldsymbol{V}_{n}-\boldsymbol{V}_{n}^{\prime}\right)(\boldsymbol{A} \boldsymbol{G})(\boldsymbol{A} \boldsymbol{G})^{\mathcal{H}}\left(\boldsymbol{V}_{n}-\boldsymbol{V}_{n}^{\prime}\right)^{\mathcal{H}}$ $\boldsymbol{X}_{n-1}^{\mathcal{H}} \boldsymbol{E}_{r}^{1 / 2}$. Since $\Delta_{e}$ is Hermitian, we can perform the following decomposition: $\Delta_{e}=\boldsymbol{U}^{\prime H} \boldsymbol{D}_{e} \boldsymbol{U}^{\prime}$, where $\boldsymbol{U}^{\prime}$ is a unitary matrix, and $\boldsymbol{D}_{e}$ is $\operatorname{diag}\left\{\lambda_{e, 1}, \lambda_{e, 2}, \ldots, \lambda_{e, L}\right\}$. Each diagonal entry $\lambda_{e, k}, k=1,2, \ldots, L$, represents an eigenvalue of $\boldsymbol{D}_{e}$. Then, the CER can be obtained by averaging Eq. 11 with respect to channel realizations $\boldsymbol{H}_{n}^{d} \boldsymbol{H}_{n}^{r}$. Let us define $h:=h^{r_{k} d} h^{s r_{k}}$, then the probability density function (PDF) of $\alpha=|h|$ is given by [10] $p_{\alpha}(\alpha)=\frac{4 \alpha}{\sigma_{r_{k} d}^{2} \sigma_{s r_{k}}^{2}} K_{0}\left(2 \sqrt{\frac{\alpha^{2}}{\sigma_{r_{k} d}^{2} \sigma_{s r_{k}}^{2}}}\right)$,

where $K_{0}(\cdot)$ is the zeroth order modified Bessel function of the second kind. By assuming each fading coefficient has unit variance, the CER can be computed by using the integration property of Bessel functions [5, Eq. 6.631.3] and follows:

$$
\begin{aligned}
\int_{0}^{\infty} x^{\theta} e^{-\alpha x^{2}} K_{\phi}(\beta x) d x= & \frac{1}{2} \alpha^{-\frac{1}{2} \theta} \beta^{-1} \Gamma\left(\frac{1+\theta+\phi}{2}\right) \\
& \times \Gamma\left(\frac{1-\phi+\theta}{2}\right) e^{\frac{\beta^{2}}{8 x}} W_{-\frac{\theta}{2}, \frac{\phi}{2}}\left(\frac{\beta^{2}}{4 \alpha}\right),
\end{aligned}
$$

where $W_{m, n}(z)=e^{-z / 2} z^{n+1 / 2} U(1 / 2+n-m, 1+2 n, z)$ is the Whittaker function with $U(\cdot, \cdot, \cdot)$ denoting confluent hypergeometric function of the second kind. By further using the approximation $U(a, 1,1 / x) \approx \ln (x) / \Gamma(a) \quad[1$, Eqs. 13.5.9] at high SNR, the CER can be simplified to

$P_{e}=P\left[\tilde{\boldsymbol{V}}_{n} \rightarrow \tilde{\boldsymbol{V}}_{n}^{\prime}\right] \leq \prod_{k=1}^{L}\left[\ln \left(\frac{\lambda_{e, k}}{8 \mathcal{N}_{0}}\right)\right]\left(\frac{\lambda_{e, k}}{8 \mathcal{N}_{0}}\right)^{-1}$.

It is worth mentioning that the CER of cooperative networks is very reminiscent of its counterpart, namely the multi-input single-output (MISO) system with co-located mutiple transmit antennas employing the DUSTC (see e.g., [13]). Their difference lies in the log term which reflects the effect of the amplification and the aggregate noise. However, this $\log$ term only leads to a coding gain loss. In fact, Eq. (15) also confirms that cooperative networks provide full diversity gain proportional to the number of relays $L$.

\section{Simulations and discussions}

In this section, we present the numerical examples and simulation results for the cooperative networks with DUSTC. We first consider CER of the system, and then the effects of relay location and comparison with the conventional system will be discussed.

\subsection{CER performance}

In Fig. 2, we plot the bounds and simulated CERs for the proposed systems when $L=1,2$, and 3 . When $L=1$, the STC-based cooperative system reduces to the conventional cooperative network, thus we can use the SER formulas derived in [4] as the CER bound of the cooperative scheme in this paper. Figure 2 shows that the bounds (Eq. 15) are tight at high SNR. However, the bounds are less accurate when SNR is low because of the log term in the analytical 


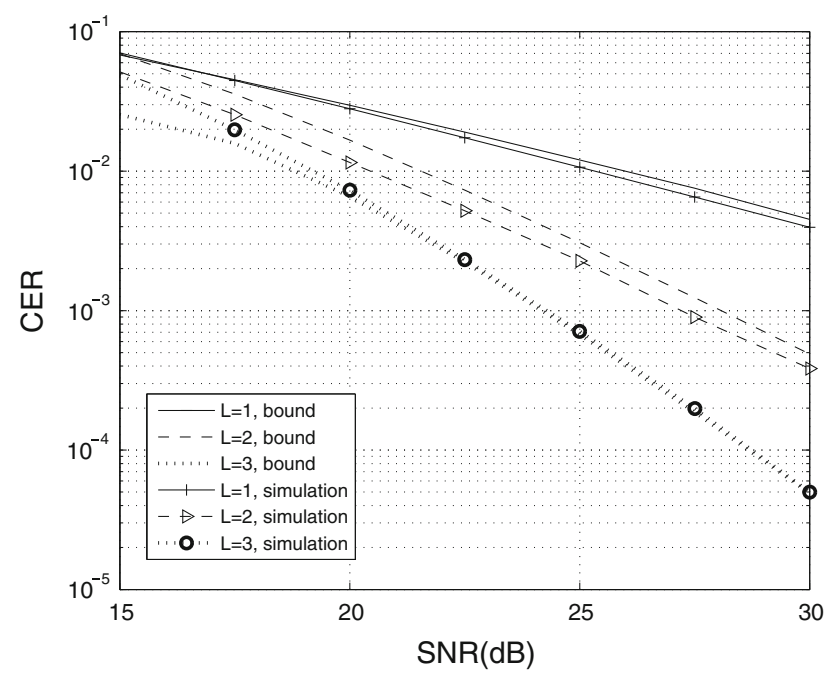

Fig. 2 The CER for AF protocol $\left(L=1,2\right.$, and 3, SNR $\left.=\bar{\gamma}_{s r_{k}}=\bar{\gamma}_{r_{k} d}\right)$

expression. It is also evident from Fig. 2 that the proposed scheme provides full diversity gain, which shows the same trend as the per-relay differential modulation with $\mathrm{AF}$ protocol in [4].

Let us consider the effect of unbalanced link quality. We assume that $\bar{\gamma}_{s r_{k}}=\bar{\gamma}_{s r}$, and $\bar{\gamma}_{r_{k} d}=\bar{\gamma}_{r d}, \forall k$, and consider (1) equal SNR for both the source-relays and relays-destination links, (2) higher SNR is assigned at the source-relays links, and (3) higher SNR is assigned at the relays-destination links with $L=1,2$ and 3. Figure 3 reveals that the coding gain can be achieved by assigning higher SNR at both links. Increasing SNR at the source-relays and relays-destination links leads to decrement of aggregate noise effect and increment SNR at the destination, respectively. Notice that the effect of SNR at the relays-destination links provides more coding gain than the source-relays links, which

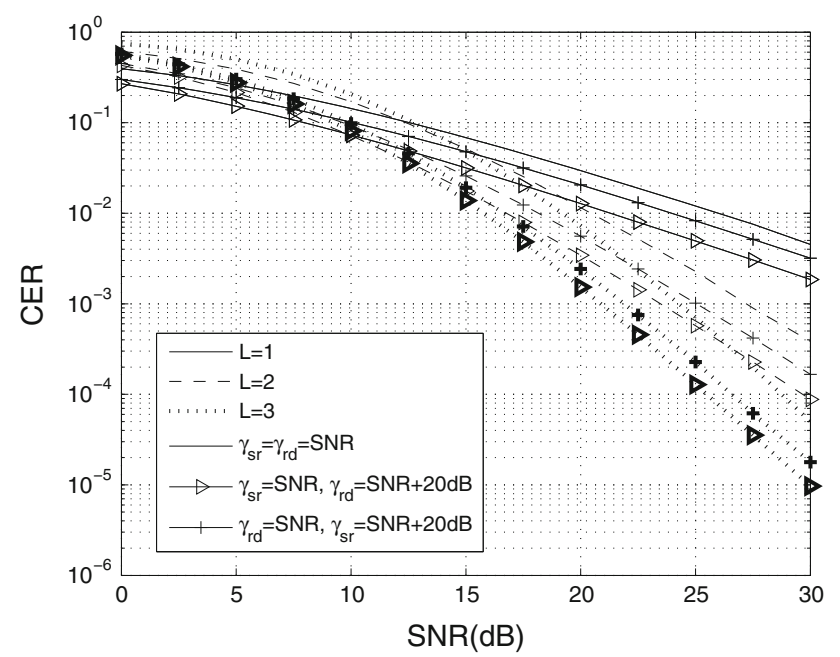

Fig. 3 The effect of unbalanced link $\operatorname{SNR}(L=1,2$, and 3) implies that the relays-destination links is more critical than the source-relays links to obtain better performance.

\subsection{Effect of relay locations}

In this subsection, we consider the effect of relay locations. We make use of the relationship between the variance of channel fading coefficient $\sigma_{i j}^{2}$ and the inter-node distance $D_{i j}$ as follows:

$\sigma_{i j}^{2}=C \cdot D_{i j}^{-v}, \quad i, j \in\left\{s, r_{k}, d\right\}$,

where $C$ is a constant and $v$ is the path loss exponent of the wireless channel. Without loss of generality, we set $C$ to 1 , and line topology is considered,i.e., $D_{s r_{k}}+D_{r_{k} d}=D_{s d}=1$. To express the energy constraint by SNR, let us define the total SNR $\rho_{T}:=\mathcal{E} / \mathcal{N}_{0}$, the transmit SNR at the source node $\rho_{s}:=\mathcal{E}_{s} / \mathcal{N}_{0}$, and the transmit SNR at the relay nodes $\rho_{r_{k}}:=\mathcal{E}_{r_{k}} / \mathcal{N}_{0}$, where we use the total energy constraint as $\rho_{T}=\rho_{s}+\sum_{k=1}^{L} \rho_{r_{k}}$.

In Fig. 4, we show the CER depending on various relay locations with $L=2$, and its corresponding contour is depicted in Fig. 5. We also plot the CER for $L=3$ with various locations in Fig. 6 . We set $v=4, \rho_{j}=10 \mathrm{~dB}$, $j \in\left\{s, r_{k}\right\}, k=1,2$ and 3. In Figs. 4 and 5, $x$ axis represents the location of relay node 1 and $y$ axis represents the location of relay node 2 . The figures show that the relay located at the same position has the minimum CER for the given one relay's location. Figure 5 reveals that the minimum CER is obtained both relays' are located at the midpoint between the source node and destination node with $L=2$. By letting $(a, b, c)$ as the location of relay node 1, 2, and 3, the CER with $L=3$ is depicted in Fig. 6 . In general, the relay located at the midpoint (0.5 in Fig. 6) enhances the error performance. Whereas, the relay located

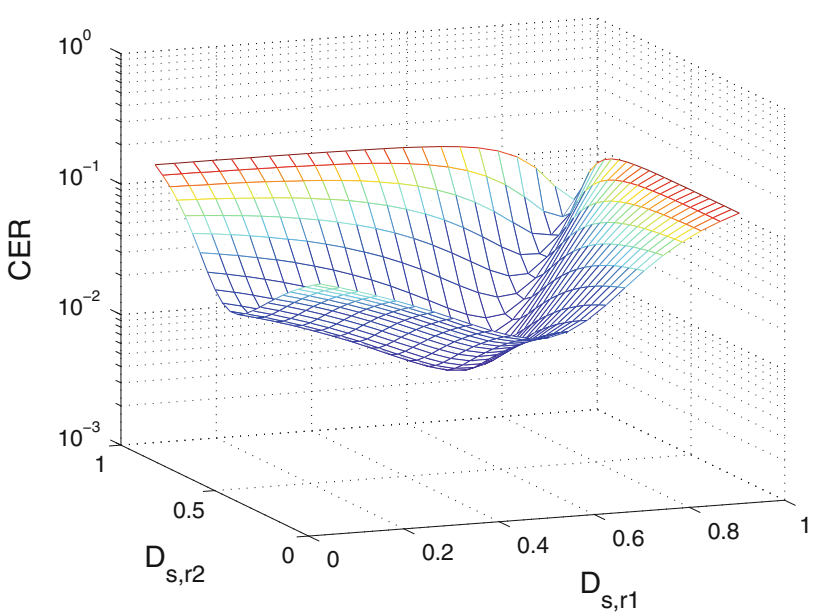

Fig. 4 CER depending on various relay locations $(L=2$, $\rho_{j}=10 \mathrm{~dB}$, where $\left.j \in\left\{s, r_{1}, r_{2}\right\}\right)$ 


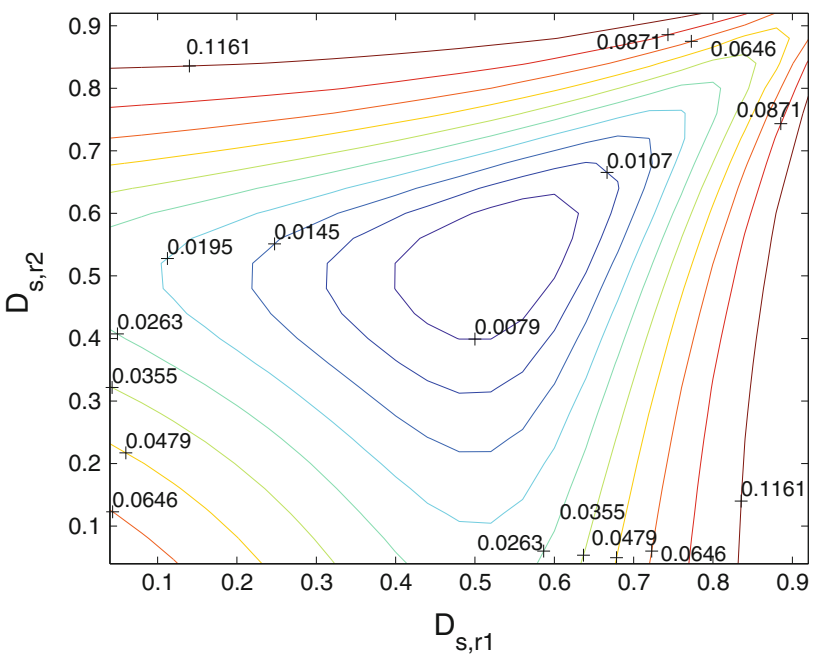

Fig. 5 Contour of CER depending on various relay locations $(L=2$, $\rho_{j}=10 \mathrm{~dB}$, where $j \in\left\{s, r_{1}, r_{2}\right\}$ )

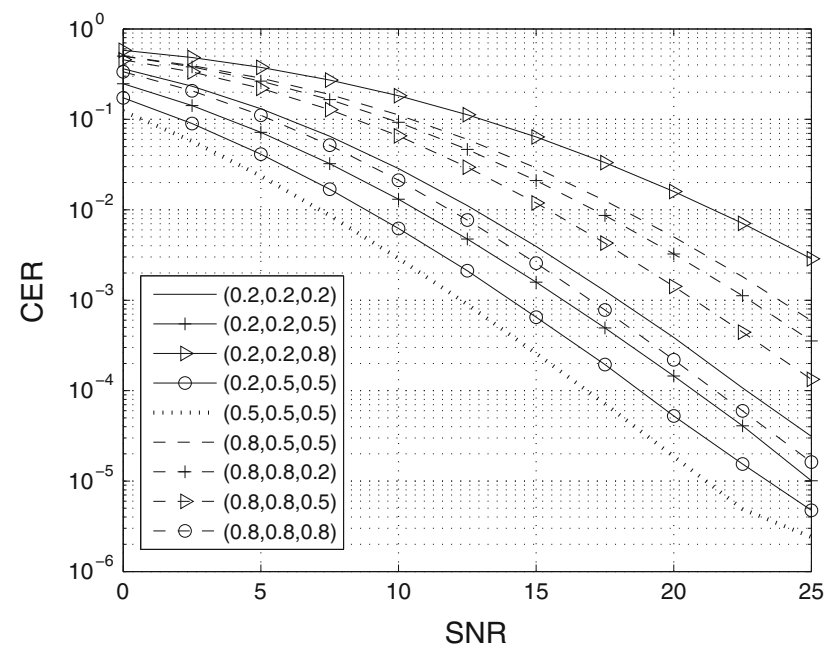

Fig. 6 CER depending on various relay locations $(L=3$, $\rho_{j}=10 \mathrm{~dB}$, where $\left.j \in\left\{s, r_{1}, r_{2}\right\}\right)$

near the destination node (0.8 in Fig. 6) induces degradation of error performance, which results in the degradation of overall error performance. Similar to the $L=2$, the system with co-located relays has better performance than the system with different relay locations. Therefore, it is good choice to select co-located relay nodes to achieve better performance. The minimum CER is obtained when all relay nodes are located at the midpoint between the source node and destination node, i.e., $(0.5,0.5,0.5)$ location.

\subsection{Effect of energy allocation}

In this subsection, the performance of the proposed system is analyzed depending on the energy (power) allocation.

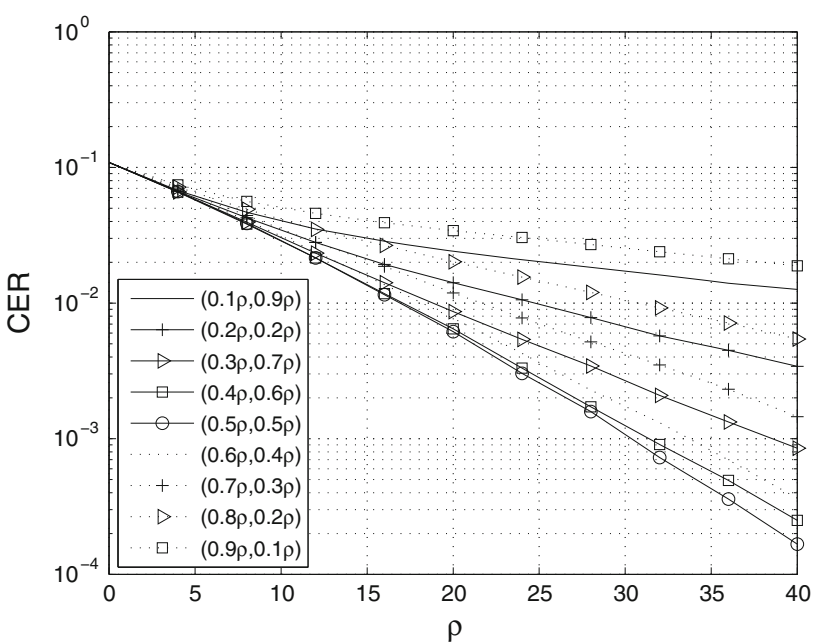

Fig. 7 CER depending on various energy allocation $(L=2,(a, b)=$ $\left.\left(\rho_{s}, \rho_{r}\right)\right)$

We consider $L=2$ and assume that each relay node has the same energy, i.e., $\rho_{r_{1}}=\rho_{r_{2}}=\rho_{r}$. The line setup, $D_{s d}=1$, is considered by locating both relay nodes at the midpoint between the source and destination node since the midpoint provides minimum error rate as we mentioned Sect. 4.2. Figure 7 depicts the CER depending on various energy allocations at the source node and relay nodes. Regardless of energy allocation, the performance has no big difference when each relay node has low energy. However, in high SNR, the equally distributed energy allocation shows minimum CER. This result is the same as the AF cooperative networks with coherent scheme in [11]. Therefore, the optimum energy allocation which minimizes the error performance is closely related to the relaying protocol not the transmission scheme such as coherent and non-coherent transmission. Based on the results of this section and Sect. 4.2, the minimum error rate can be obtained by locating relay nodes at the midpoint between the source node and destination node and assigning equal energy.

\subsection{Comparison with the conventional system}

It is worth noting the differences between our schemes and the conventional cooperative networks. For the conventional system as in $[8,4,17]$, the signal is transmitted by repetitive manner. Specifically, during the first time slot, the source broadcasts the information symbol to the relays, and then the $L$ relays forward their amplified information to the destination using distinct channels at the relays-destination links. If the original information symbols of both the STC-based and conventional cooperative networks are equi-probable binary signal $(\eta=1$ in 


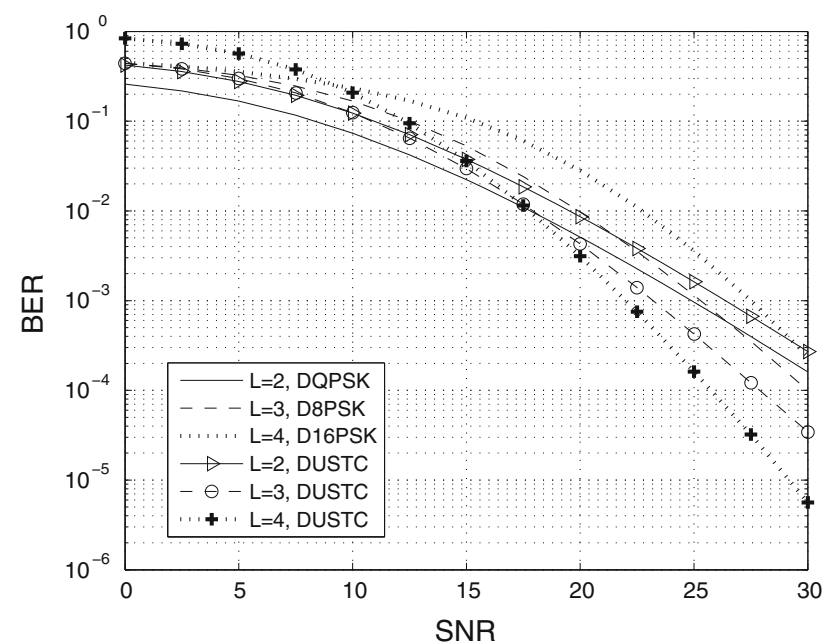

Fig. 8 BER comparison between the conventional systems and STCbased systems with same modulation size $\left(\mathrm{SNR}=\bar{\gamma}_{s r_{k}}=\bar{\gamma}_{r_{k} d}\right)$

this paper), the STC-based system uses $2 L$ time slots for $L$ symbols transmission; whereas the conventional system uses $(L+1)$ time slots for 1 symbol transmission. Hence, the STC-based system can provide higher data rate especially when $L>1$. This implies that the STC-based system can potentially provide the differential benefit as well as high transmission rate regardless of the number of relays. On the other hand, conventional system also can support higher data rate by increasing modulation size of the data symbol, but this causes error performance loss. To capture this phenomenon, we compare the bit error rate (BER) of the conventional system which adopts the same modulation size with the STC-based system in Fig. 8, i.e., use DQPSK, D8PSK, and D16PSK for $L=2,3$, and 4 , respectively, for the conventional system. Notice that the performance is compared using BER not CER. For BER comparison, we convert CER of the DUSTC system to BER. Then, the data rate of each modulation is $L /(L+1)$ bps, whereas the data rate of STC-based system is $1 / 2$ bps regardless of $L$. The figure shows that STC-based system performs comparably with or better than the conventional system especially when $L \geq 3$. For the conventional system, the error performance decreases as $L$ increases. However, for the STC-based system, the BER is not affected dramatically regardless of $L$, and the diversity gain is always guaranteed.

\section{Conclusions}

In this paper, we explored the performance of cooperative networks employing the differential unitary space time code (DUSTC) with amplify-and-forward (AF) protocol.
Based on the DUSTC-based transmission scheme, the codeword error rate (CER) bound was analyzed under high SNR assumption. Our analysis and simulations showed that: (1) AF protocol provides full diversity gain; (2) colocated relay selection is good choice to achieve good error performance; and (3) the minimum CER can be obtained by locating relay nodes at the midpoint between the source node and destination node and by distributing the energy equally in each node. The comparison with conventional cooperative networks revealed that STC-based cooperative networks can support higher data rate by providing comparative error performance compared with the conventional system.

Acknowledgments This work is in part supported by the IT R\&D program of MKE/IITA [2007-F-039-01, Vehicle Multi-hop Communication Technology Development]. Part of the results in this paper was presented at IEEE Conference on Acoustics, Speech and Signal Processing, Las Vegas, Nevada, 2008.

Open Access This article is distributed under the terms of the Creative Commons Attribution Noncommercial License which permits any noncommercial use, distribution, and reproduction in any medium, provided the original author(s) and source are credited.

\section{References}

1. Abramowitz, M., \& Stegun, I. A. (1972). Handbook of mathematical functions with formulas, graphs, and mathematical tables. Dover: New York.

2. Cao, R., \& Yang, L. (2008). Practical issues in resource optimization of relay networks. In Proceedings of CISS.

3. Cho, W., \& Yang, L. (2008). Optimum resource allocation for relay networks with differential modulation. IEEE Transactions on Communications, 56(4), 531-534.

4. Cho, W., Cao, R., \& Yang, L. (2008). Optimum resource allocation for amplify-and-forward relay networks with differential modulation. IEEE Transactions on Signal Processing, 56(11), $5680-5691$.

5. Gradshteyn, I. S., \& Ryzhik, I. M. (2000). Table of integradls, series, and products (6th ed.). New York: Academic Press.

6. Hochwald, B. M., \& Sweldens, W. (2000). Differential unitary space-time modulation. IEEE Transactions on Communications, 48(12), 2041-2052.

7. Jing, Y., \& Jafarhani, H. (2008). Distributed space-time coding for wireless relay network. IEEE Transactions on Communications, 56(7), 1192-1100.

8. Laneman, J. N., \& Wornell, G. W. (2000). Energy-efficient antenna sharing and relaying for wireless networks. In Proceedings of wireless communications and networking conference (Vol. 1, pp. 7-12).

9. Oggier, F., \& Hassibi, B. (2007). A coding scheme for wireless networks with multiple antenna nodes and no channel information. In Proceedings of international conference on acoustics, speech and signal processing (Vol. 3, pp. 413-416).

10. Patel, C. S., Stüber, G. L., \& Pratt, T. G. (2006). Statistical properties of amplify and forward relay fading channel. IEEE Transactions on Vehicular Technology, 55(1), 1-9. 
11. Ribeiro, A., Cai, X., \& Giannakis, G. B. (2005). Symbol error probabilities for general cooperative links. IEEE Transactions on Wireless Communications, 4(3), 1264-1273.

12. Tarokh, V., Seshadri, N., \& Calderbank, A. R. (1998). Space-time codes for high data rate wireless communication: Performance criterion and code construction. IEEE Transactions on Information Theory, 44(2), 744-765.

13. Yiu, S., Schober, R., \& Lampe, L. (2006). Distributed space-time blcok coding. IEEE Transactions on Communications, 54(7), 1195-1206

14. Yu, M., Li, J., \& Sadjadpour, H. (2005). Amplify-forward and decode-forward: The impact of location and capacity contour. In Proceedings of MILCOM (Vol. 3, pp. 1609-1615).

15. Wang, G., Zhang, Y., \& Amin, M. (2006). Differential distributed space-time modulation for cooperative networks. IEEE Transactions on Wireless Communications, 5(11), 3097-3180.

16. Wang, T., Yao, Y., \& Giannakis, G. B. (2006). Non-coherent distributed space-time processing for multiuser cooperative transmissions. IEEE Transactions on Wireless Communications, 5(12), 3339-3343.

17. Zhao, Q., \& Li, H. (2007). Differential modulation for cooperative wireless systems. IEEE Transactions on Signal Processing, 55(5), 2273-2283.

\section{Author Biography}

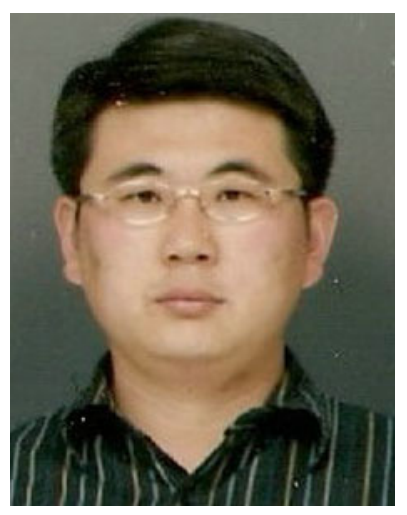

Woong Cho received the B.S. degree in electronics engineering from the University of Ulsan, Ulsan, South Korea, in 1997, the M.S. degrees in electronic communications engineering and electrical engineering from Hanyang University, Seoul, South Korea, in 1999, and the University of Southern California, Los Angeles, in 2003, respectively, and the $\mathrm{Ph} . \mathrm{D}$. degree in electrical and computer engineering from University of Florida, Gainesville, in 2007. He is currently with the Electronics and Telecommunications Research Institute (ETRI), Daejeon, South Korea. His research interests are cooperative/relay networks, vehicular communications, telematics, and signal processing for wireless communications. 